\title{
The Relationship between Cognitive Therapeutic Exercises and Basal Ganglia Function in Patients with Basal Ganglia Region Stroke; a Pilot Study
}

\author{
Sang-Mi Joung ${ }^{1}$, Byung-Il Yang ${ }^{*}$ \\ 'Dept. of Occupational Therapy, Sangji Youngseo University, Gangwon-do, Republic of Korea \\ ${ }^{* 2}$ Dept. of Physical Therapy, Sangji University, Gangwon-do, Republic of Korea
}

Purpose This study was aimed that the cognitive therapeutic exercises (CTE) benefit on motor function in patients with basal ganglia stroke. Methods The subjects assigned to a right hemiplegia group (RHG) and left hemiplegia group (LHG) and performed the CTE 5 times a week for 4 weeks. Manual functional test (MFT), Korean-Modified Barthel Index (K-MBI) and Sensory Function Test (SFT) were used to assess motor functions of basal ganglia. Results There were significant differences in MFT in non-hemi side (MFT_nh), K-MBI and SFT in the RHG and in K-MBI in the LHG. Conclusion The subjects with the RHG more were improved in most assessments than that of theLHG. As a whole, the CTE influenced the basal ganglia functions.

Key words Basal ganglia, Cognitive therapeutic program, Manual functional test, Korean-Modified Barthel Index, Sensory Function Test

Corresponding author Byung Il Yang (bobathyang@naver.com)

Received date 09 January 2019

Revised date 29 January 2019

Accepted date 07 February 2019

\section{Introduction}

Sensory disorder disturbs correct movements and diminishes sensory inputs and movements with feedback. Patients with sensory disordershesitate to move their bodies actively and the movements are not coordinated and accurate. ${ }^{1)}$ Patients with basal ganglia infarction show commonly cognitive impairments. The basal ganglia strokes have shown not only restriction of motor function historically but also a variety of cognitive function impairments. Moreover, the impairment of memory, learning and visuospatial skills were related to basal ganglia stroke, which was ascribed to the problem of cerebello-basalganglio-thalamo-cortical loops. ${ }^{2)}$ The Cognitive therapeutic exercises (CTE) focus on reorganization of central nervous system by learning, which causes motor function recovery. Motor function recovery is related closely to cognitive process and activation of the brain such as awareness, attention, memory, judgement and language. And, the quality of the recovery depends on how well these

http:dx.doi.org/10.17817/2019.01.29.111354 cognitive elements have done together. ${ }^{3)}$ There are four principles which are very significant on the CTE. First one is attention. It is important to make patients pay attention on the CTEto increase therapeutic effects and reorganize motor function. Second, make them pay attention on somatosensory information with eye closed. Third, general and other therapeutic tools should be used to solve cognitive problems through interactions between circumstances and human body. Fourth, do not force patients to contract muscles for proper motor units recruited. The purpose of the CTE is not to teach varied postures, movements and physical performance postures specifically to the patients but to improve the ability of organization of the spatial, temporal and amplitude elements in the sequence of the exercises as much as possible through interactions between circumstances and human body. ${ }^{4)}$ In previous study, there were improvement in manual function test (MFT), manual muscle test (MMT) and box and block test (BBT) after having applied the CTE to upper extremity of the patients with stroke 30 minutes per session for 8 weeks." Therefore, basal 
ganglia stroke patients need specific therapeutic exercises for motor functions which can be the exercises combined with cognitive aspects. In this study, the CTE would benefit on motor functions in patients with basal ganglia stroke.

\section{Material and Methods}

This study was conducted from August $6^{\text {th }}$ to $31^{\text {st }}$, 2017 at I hospital in Ilsan, South Korea by an occupational therapist with 5 years of clinical experiences. Eight in-patients who got more than 19 points in mini mental state examination - Korean version (MMSE-K) were assigned into a right hemiplegia group (RHG, $\mathrm{n}=4$ ) and let hemiplegia group (LHG, n=4) in this study and performed the CTE for 30 minutes per session, 5 times a week for 4 weeks. The subjects were known on the purpose, duration and methods of the research, and they submitted the informed consent in advance. Including criteria are as follows: (1) more than 6 months since onset of stroke, (2) more than 19 points in MMSE-K, (3) no orthopedic problem in the upper limb. The subjects were assessed by Manual functional test (MFT), Korean-Modified Barthel Index (K-MBI) and Sensory Function Test (SFT) in pre and post-test.

MFT consists of 4 items in upper limb exercise, 2 items in grasping and 2 items in finger manipulation. It was developed to apply easily and objectively to patients and shows the upper limb function recovery and the functional level of the activities of daily living (ADL). MFT's inter-rater and intra-rater reliabilities and internal validity were 0.95 points of Cronbach's $\alpha$ in the previous study. ${ }^{6}$ K-MBI consists of 7 items of self-care and 3 items of mobility index, a total of 10 items in ADL. Higher scores present independence in ADL. ${ }^{7}$

SFT was performed with a total of 7 different sensory input on hemiplegic arm such as pain, tactile, pressure, heat, kinesthesis, stereognostic sense, 2-points discrimination.

If a patient did not know what kind of the stimulate was given and where the stimulate was given to in each items, it graded 0 point. If the patient knew ei- ther a kind of stimuli or location, the patient got 1 point. And if the patient knew both of them, the patient got 2 points. As a whole, a total of 14 points in 7 items was a perfect score. The subjects were performed the test 10 times and then if they knew more than 7 times out of 10 times, sensory function was regarded as intact. CTE consisted of 4 different exercises.

\section{Cognitive training for shoulder joint through motor imagery ${ }^{8)}$}

This training is one of the spatial tasks applying to the shoulder joint to control the shoulder joint through motor imagery of the shoulder joint. The imagery is used to improve an ability of perception of the shoulder movement in flexion and abduction. A therapist has a patient tell the feeling of flexion and abduction of the shoulder joint in non-affected side in sitting on a chair without leaning on the back rest. The therapist moves the patient's shoulder in affected side passively and make the patient tell the feeling of it, and makes the patient move the affected arm with the imagery of the non-affected arm then let the patient present his/her feeling of the movement. After presenting the feeling of the active-assistive flexion and abduction of the shoulder joint in affected side, let the patient move his/her non-affected shoulder joint in flexion and abduction as comparing the movement of the affected arm. Let the patient present of the sensory imagery felt during the shoulder exercises in affected and non-affected sides. Make the patient compare the difference of the sensory imageries between in affected and non-affected sides. Therefore, the patient can pay attention on the shoulder joint as using the sensory imagery and the shoulder movement.

\section{Perception training of the shoulder and elbow joint angles using circular orbits ${ }^{3)}$}

This is a training to distinguish the angles of the shoulder and elbow joints using circular orbits as a spatial task. This training was performed to perceive the shoulder joint movements and distinguish difference in distances of the circles on a plate of circular orbit. A patient sits on a chair in front of a desk with- 
out leaning on the back rest. Have the patient take a look at the plate of the circular orbits to analyze the different sizes of circles on it. And then, the therapist makes the patient's arm moved passively to provide his/her the information of the location of the orbits with eyes closed. Ask him/her about the distances and let him/her explain it verbally.

\section{Compression perception training of the forearm and wrist joint using sponges ${ }^{5}$}

This is a training to perceive differences in compression between the forearm and wrist joint as a tactile task. Sponges are used to improve on recognition of slight different compression. Make the subject sit on a chair without leaning on the back rest and then the training is performed by using 3 different sponges which are same in height but different in hardness. The therapist has the patient press the sponge down with eye closed. Make him/her pay attention on the surface of the sponges attached with the hand. Ask him/her to recognize differences of the sponges in hardness. The subjects pay attention on not the sponges but one of the joints which are shoulder joint, forearm and wrist joint. Ask him/her to recognize differences of the locations and hardness of the sponges.

\section{Finger perception training using a tactile plate $^{3)}$}

This is a task to recognize differences of the materials in the tactile plate. It is performed to distinguish differences of the superficial surface of the materials and perceive the finger movements. A subject sits on a chair without leaning on the back rest of it and put the both arms on a table. Five different tactile plates with soft or tough materials should be prepared for the subjects to provide visual information and the tactile plate to affected and non-affected sides. The therapist moves his/her fingers passively to touch the surface of the materials and then ask if he/she can recognize differences of the surface of the materials and the finger movements. This study was analyzed using SPSS 18 version. The general characteristics was analyzed with chi-square test. The difference of pre and post-test in the RHG and LHG was analyzed with Wilcoxon signed ranks test. $\alpha=.05$ level of significance was used for the statistical test.

\section{Results}

\section{General characteristics of the subjects}

There are 4 subjects in the RHG and LHG each. Two males and 2 females, mean age of $68.7 \pm 8.5$ years old, mean onset of $18.2 \pm 8.7$ month and mean MMSE-K of $25.2 \pm 4.5$ point are in the RHG. And, two males and 2 females, mean age of $62.5 \pm 16.7$ years old, mean onset of $24.7 \pm 4.5$ month and mean MMSE-K of $26.0 \pm 2.9$ point are in the LHG. (Table 1) There are significant differences in MFT_nh, KMBI and SFT in the RHG and in KMBI in the LHG. (Table 2)

\section{Discussion}

Table 1. General characteristics of the subjects $(\mathrm{N}=8)$

\begin{tabular}{llcc}
\hline & & RHG (n=4) & LHG (n=4) \\
\hline \multirow{2}{*}{ Gender } & Male & $2(50 \%)$ & $2(50 \%)$ \\
Age (years) & Female & $2(50 \%)$ & $2(50 \%)$ \\
Onset (month) & & $68.7 \pm 8.5$ & $62.5 \pm 16.7$ \\
MMSE-K (point) & & $18.2 \pm 8.7$ & $24.7 \pm 4.5$ \\
Stroke & Hemorrhage & $25.2 \pm 4.5$ & $26.0 \pm 2.9$ \\
& Infarction & $2(50 \%)$ & $2(50 \%)$ \\
\hline
\end{tabular}

n (\%) / M \pm SD; RHG, Right hemiplegia group; LHG, Left hemiplegia group; MMSE-K, Mini mental state examination - Korean 
Table 2. Variations of basal ganglia function in pre and post-test $(\mathrm{N}=8)$

\begin{tabular}{ccccccc} 
& \multicolumn{3}{c}{ RHG $(\mathrm{n}=4)$} & & \multicolumn{2}{c}{ LHG $(\mathrm{n}=4)$} \\
& pre & post & $p$ & pre & post & $p$ \\
\hline MFT_h & $10.0 \pm 10.7$ & $10.8 \pm 10.8$ & 0.29 & $5.3 \pm 3.2$ & $5.5 \pm 2.1$ & 0.39 \\
MFT_nh & $28.5 \pm 0.6$ & $29.3 \pm 1.0$ & $0.04^{*}$ & $30.0 \pm 1.2$ & $30.5 \pm 0.6$ & 0.07 \\
KMBI & $45.8 \pm 11.9$ & $50.0 \pm 14.3$ & $0.05^{*}$ & $60.5 \pm 22.6$ & $67.8 \pm 15.9$ & $0.03^{*}$ \\
SFT & $7.3 \pm 2.5$ & $8.8 \pm 2.2$ & $0.03^{*}$ & $8.0 \pm 4.1$ & $8.5 \pm 3.5$ & 0.07 \\
\hline
\end{tabular}

$\mathrm{M} \pm \mathrm{SD} ; *$, $P<0.05$; RHG, Right hemiplegia group; LHG, Left hemiplegia group; MFT $h$, Manual functional test in hemi side; MFT_nh, Manual functional test in non-hemi side; KMBI, Korean-Modified Barthel Index; SFT, Sensory Function Test

The main role of the CTE is to bring the widespread recovery back in the brain as making a patient with stroke be able to control motor functions, which is cognitively affecting somatosensory area of the brain on reorganizing activities. ${ }^{9}$ In the previous study, the subjects performed the exercises as comparing the pressures putting on the sponges in the shoulder and elbow joints. As a result, MFT improved from 23 to 29 points in the left hand and from 20 to 25 points in the right hand as well as improvement in perceiving the joint angles. ${ }^{8)}$ In addition, in the previous study, after performing pressure perception training using sponges and the spatial perception training using circular orbits, muscle tone in the affected arm was improved by $23.3 \%$ and MFT was improved by 9.37 $\%{ }^{10)}$ Moreover, in the previous study, the subjects have performed the tactile task training using sponges in the affected side and the cognition therapeutic treatment as a spatial task using figure panels for 4 weeks. As a result, MBI was improved by 31 points and MFT was improved from 3 to 9 points. ${ }^{11)}$ What is more, another previous study revealed that after the training using sponges, Semmes-Weinstein Monofilament which is an assessment tool for sensory function was improved from 6.65 to $3.22 \mathrm{~mm}^{12)}$ In this study, the CTE was applied to the patients with basal ganglia stroke for 4 weeks. Basal ganglia play a significant role in programming motor activities and it is polished by experiences so that the movement becomes more efficient and accurate. To improve more sophisticated movement, sensory information is significantly important in terms of body schema. Since the CTE focuses on tactile input as well as proprio- ception, with these accurate information, the brain takes part more actively in motor programming especially. The basal ganglia provide more a time-saving and efficient movement strategy. The results of this study showed improvement somewhat to significantly in all assessments. What that means is that the subjects have had chances to reorganize body schema and sequence of the movement to correct the wrong movements by a trial-and-error since the intervention started. As a result, the subjects became to be able to choose the best strategy in a certain situation. As shown in the results of each assessment, the subjects with right hemiplegia more improved all assessments than that of left hemiplegia patients. Since the right side brain takes care of spatial movement, the subjects with left hemiplegia showed less improvement in most assessments. As a whole, the CTE aiming at sensory integration, movement sequences and memory influenced positively the basal ganglia resulting in improvement in sensory and functional movements. This study was conducted as a pilot study with a small number of subject so that the future study should have more participants to generalize the results.

\section{References}

1. Trombly CA. Occupational therapy for physical dysfunction. 2002:1-15.

2. Zuo LJ, Li ZX, Zhu RY, et al. The Relationship between Cerebral White Matter Integrity and Cognitive Function in Mild Stroke with Basal Ganglia Region Infarcts. Scientific reports. 2018;8(1):8422.

3. Jang JY, Kim JM. The Effects of Cognitive Therapy Exercise on the Affected Upper Extremity Function and 
Activities of Daily Living for Stroke Patient. Journal of Korean Society of Neurocognitive Rehabilitation 2012;4:55-65.

4. Lee SA, Lee HS. Practical and Methodological New Cognitive Exercise Therapy. Rehabilitation Research Institute affiliated with RI Korea. 2005;9(2):142-64.

5. Kim YS. Effect of Cognitive Therapeutic Exercise on Improvement Hand Function With Hemiplegic Patient After Stroke. Journal of Korean Society of Neurocognitive Rehabilitation. 2009;1(1):11-24.

6. Miyamoto S, Kondo T, Suzukamo Y, et al. Reliability and validity of the Manual Function Test in patients with stroke. Am J Phys Med Rehabil. 2009;88(3):247-55.

7. Shah S, Vanclay F, Cooper B. Improving the sensitivity of barthel Index for strokerehabilitation. Journal of Clinical Epidemiology. 1989;42(8):703-9.

8. Choi JE, Lee SS. Effect of Stretch Reflex Control of the Upper Limb on Recovery Hand Function With Stroke Patient. Journal of Korean Society of Neurocognitive Rehabilitation. 2009;1(1):75-86.
9. Pedretti LW, Early, M. B. Occupational therapy: Practice skills for physical dysfunction. 2001:3-12. London: Mosby.

10. Kim MY, Lee TY. Effect of Tactile and Spacious Cognitive Task Training on Decrease of Spasticity With Hemiplegic Patient. Journal of Korean Society of Neurocognitive Rehabilitation. 2010;2(1):56-66.

11. An SN, Lee JW. Effect of Cognitive Therapeutic Exercise on Recovery of the Upper Limb Function in hemiplegia Journal of Korean Society of Neurocognitive Rehabilitation. 2009;1(1):43-55.

12. Lee SA. Effect of Cognitive Therapeutic Exercise on Improvement Sensory Perception with Hemiplegic Patient after Stroke. The Journal of Occupational Therapy for the Aged and Dementia. 2010;4(1):47-55. 
